\title{
Acculturation and Subclinical Atherosclerosis among U.S. South Asians: Findings from the MASALA study
}

\author{
Kanaya $\mathrm{AM}^{* 1}$, Ewing $\mathrm{SK}^{1}$, Vittinghoff $\mathrm{E}^{1}$, Herrington $\mathrm{D}^{2}$, Tegeler $\mathrm{C}^{2}$, Mills $\mathrm{C}^{1}$ and Kandula $\mathrm{NR}^{3}$ \\ ${ }^{1}$ University of California, San Francisco \\ ${ }^{2}$ Wake Forest University Medical Center \\ ${ }^{3}$ Northwestern University
}

*Corresponding author: Kanaya AM, University of California, San Francisco, Box 0320, 1545 Divisadero Street, Suite 311, San Francisco, CA 94143-0320, Fax: (415) 514-8666, Tel: (415) 353-7919, E-mail: alka. kanaya@ucsf.edu

Citation: Kanaya AM, Ewing SK, Vittinghoff E, Herrington D, Tegeler C, et al. (2014) Acculturation and Subclinical Atherosclerosis among U.S. South Asians: Findings from the MASALA study. J Clin Exp Res Cardiol 1(1): 102. doi: 10.15744/2394-6504.1.102

Received Date: June 07, 2014 Accepted Date: June 16, 2014 Published Date: June 23, 2014
Abstract
Objective: Longer duration of residence among immigrants to the United States, a proxy measure of acculturation, has been associated with higher subclinical atherosclerosis. South Asian immigrants are the second fastest growing immigrant group in the U.S. but little is known about the effects of acculturation with atherosclerosis in this high cardiovascular risk population.
Methods: We conducted a cross-sectional analysis using data from a community-based cohort called the Mediators of Atherosclerosis in South Asians Living in America (MASALA) study. Participants $(n=900)$ were between ages of 40-84 years and had no existing cardiovascular disease. We developed a multi-dimensional measure of acculturation in South Asians, called traditional cultural beliefs, and measured other proxy measures of acculturation to determine whether they were associated with higher levels of subclinical atherosclerosis after controlling for socioeconomic, behavior/lifestyle, and cardiovascular risk factors.
Results: Mean duration of residence in the U.S. was $27 \pm 11$ years and tertiles of strength of traditional cultural beliefs were examined. Longer duration of U.S. residence was associated with higher levels of coronary artery calcium even after adjustment for covariates and lifestyle mediators. The novel measure of strength of traditional cultural beliefs was associated with lower common carotid intima media thickness among those with moderate traditional beliefs only.
Conclusions: These findings support the need for better conceptualization and measurement of how migration influences cultural beliefs and practices, and their subsequent influence on health behaviors and cardiovascular disease risk.
Keywords: Atherosclerosis; Acculturation; Athnicity
List of Abbreviations: BMI-Body mass index; CAC-Coronary artery calcium; CI-Confidence interval; cIMT-Carotid intima-media thickness; CT-Computed tomography; HDL-High density lipoprotein; LDL-Low density lipoprotein; MASALA- Mediators of Atherosclerosis in South Asians Living in America; NWU-Northwestern University; RRR-Relative risk ratio; SHARE- Study of Health Assessment and Risk in Ethnic groups; UCSF-University of California, San Francisco; US-United States

\section{Introduction}

South Asian immigrants (individuals from India, Pakistan, Bangladesh, Sri Lanka and Nepal) comprise the second largest Asian subgroup in the United States, having grown by $81 \%$ between Census 2000 and 2010, with approximately 3.4 million U.S. residents currently [1]. Similar to other immigrants, South Asians undergo cultural changes after migration to the U.S. "Acculturation" has been defined as a process where members of one group adopt the attitudes, values, customs, beliefs and behaviors of another culture $[2,3]$. Acculturation to the U.S. in other immigrant groups such as Japanese and Mexicans has been shown to adversely affect health behaviors and lead to higher rates of obesity, diabetes, hypertension and heart disease [4,5]. Longer duration of residence in the U.S. among immigrants, a surrogate marker for acculturation, has also been associated with higher levels of subclinical atherosclerosis among Latinos, African Americans and Chinese Americans in the Multi-Ethnic Study of Atherosclerosis (MESA) [6-8]. Very few studies have measured acculturation and its association with atherosclerosis or cardiovascular disease among U.S. South Asians. 
Literature on the South Asian diaspora suggests that South Asian immigrants retain a strong sense of cultural and ethnic identity $[9,10]$. Anthropologists working with migrant groups have sometimes pointed to a persistence of cultural differences [11], which contrasts with ideas about acculturation. In particular, South Asians have been noted to hold onto core values and practices at home (e.g. eating South Asian food, traditional family structure) while easily adapting to interactions, language, and etiquette outside the home [12,13]. These findings suggest that the traditional proxy measures of acculturation which have been used in epidemiologic research, such as years in the U.S. or English language use [14], may not adequately capture the multidimensionality of culture and acculturation on the health of South Asian immigrants.

We previously developed a scale to measure traditional cultural beliefs using qualitative methods [15] and have tested it in a pilot study of Asian Indians [16]. We administered this traditional cultural beliefs scale and other demographic, communication, social interactions, and dietary practice questions to capture specific domains of acculturation in a large community-based cohort of U.S. South Asians. We aimed to 1) determine associations between different measures of acculturation and subclinical atherosclerosis among U.S. South Asians, and 2) investigate whether the association between the traditional cultural beliefs scale is stronger than the proxy measures of acculturation for atherosclerosis. We determined whether this more comprehensive set of measures of acculturation were associated with higher levels of subclinical atherosclerosis after controlling for socioeconomic, behavior/ lifestyle, and cardiovascular risk factors. We hypothesized that the traditional cultural beliefs scale which is linked to specific behaviors would be more closely associated with atherosclerosis outcomes rather than other proxy measures of acculturation.

\section{Material and Methods}

\section{Study sample}

The MASALA study is a community-based cohort of South Asian men and women from two clinical sites (San Francisco Bay Area at the University of California, San Francisco (UCSF) and the greater Chicago area at Northwestern University (NWU)). All visits were conducted by trained bilingual study staff and all consent forms were translated into Hindi and Urdu. The baseline examination was conducted from October 2010 through March 2013, and a total of 906 participants were enrolled. The institutional review boards of UCSF and NWU approved the MASALA study protocol.

\section{Eligibility Criteria}

Study methods have been previously reported [17]. Briefly, to be eligible for the MASALA study, participants had to (1) selfidentify with South Asian ethnicity, (2) be between ages of 40 and 84 years, and (3) be able to speak and/or read English, Hindi or Urdu, the main languages spoken by immigrants from South Asia. We used identical exclusion criteria to MESA [18] which included having a physician diagnosed heart attack, stroke or transient ischemic attack, heart failure, angina, using nitroglycerin, or having had a cardiovascular procedure such as coronary artery bypass graft surgery, angioplasty, valve replacement, pacemaker or defibrillator implantation, or any surgery on the heart or arteries. Those with current atrial fibrillation or in active treatment for cancer were excluded. Those with life expectancy $<5$ years due to a serious medical illness, impaired cognitive ability as judged by the reviewer, plans to move out of the study region in the next 5 years, or those living in a nursing home or waiting to enter a nursing home were also excluded. Due to CT scanner limitations, those weighing over $300 \mathrm{lbs}$. were excluded.

\section{Traditional Cultural Beliefs and other Acculturation measures}

Since there are no validated multidimensional instruments to measure acculturation among South Asians, we attempted to capture several specific domains of acculturation including language use and fluency, social connections, dietary preferences, and cultural beliefs. We also collected data on nativity, years of residence in the U.S., and calculated percent of life spent in the U.S. (years of residence in the U.S./age).

Using data from prior qualitative work in Asian Indians [15], we created a traditional cultural beliefs scale consisting of 7 items. The base question was "How much would you wish these traditions from South Asia would be practiced in America?" The seven items included: performing religious ceremonies; serving sweets at ceremonies; fasting on specific occasions; living in a joint family; having an arranged marriage; eating a staple diet of chapattis, rice, daal, vegetable, and yogurt; using spices for health and healing. The items were scored on a Likert scale with higher scores representing weaker traditional Indian beliefs. Preliminary description of this continuous scale showed a U-shaped association with cIMT outcomes. Therefore, we analyzed tertiles of the score ( $<12$ for strongest, $12-17$ for moderate, and $\geq 17$ representing weakest traditional beliefs). The Cronbach's alpha for this scale in the current study sample was 0.83 with similar reliability in both men and women.

\section{Covariates}

We gathered information on participant's demographic data (sex, age, and clinical site). These variables were included in all multivariate models. 


\section{Potential mediators}

Mediators considered were total caloric intake, total physical activity, exercise, tobacco and alcohol use, waist circumference, fasting glucose, systolic blood pressure, LDL-cholesterol, HDL-cholesterol, and cholesterol lowering medication use which have all been associated with atherosclerosis in this study population [19].

Total caloric intake over the previous year was assessed using the Study of Health Assessment and Risk in Ethnic Groups (SHARE) food frequency questionnaire, which was created and validated among South Asians in Canada [20]. Total physical activity and total exercise in MET-min/week were assessed using the Typical Week's Physical Activity Questionnaire [21]. Total physical activity included intentional exercise, occupational activities, volunteer activities, household chores, yardwork, child/adult care, transportation, and leisure activities. Intentional exercise included walking for exercise, dancing, team sports, dual sports, individual activities, moderate conditioning activities, and heavy conditioning activities. Tobacco use and alcohol consumption were determined by questionnaires.

Seated resting blood pressure was measured three times using an automated blood pressure monitor (V100 Vital sign monitor, GE Medical Systems, Fairfield, CT) and the average of the last two readings used for analysis. Hypertension was defined as self-reported treatment for hypertension or a systolic blood pressure $\geq 140 \mathrm{mmHg}$ or diastolic blood pressure $\geq 90 \mathrm{mmHg}$. Participant weight was measured on a standard balance beam scale or digital weighing scale and height using a stadiometer. Waist circumference was measured using a flexible tape measure tape at the site of maximum circumference midway between the lower ribs and the anterior superior iliac spine.

After a requested 12 hour fast, blood tests were obtained. Fasting plasma glucose was measured by the glucose oxidase method; total cholesterol, triglycerides, and high-density lipoprotein (HDL) cholesterol were measured by enzymatic methods (Quest, San Jose, CA) and low-density lipoprotein (LDL) cholesterol was calculated [22]. Diabetes was classified if a participant was using a glucose-lowering medication or had a fasting plasma glucose $\geq 126 \mathrm{mg} / \mathrm{dl}$. Impaired fasting glucose was defined for those with fasting glucose between 100-125 mg/dl, and normal glucose was $<100 \mathrm{mg} / \mathrm{dl}$ [23]. Fasting serum samples were batched for insulin measured by the sandwich immunoassay method (Roche Elecsys 2010, Roche Diagnostics, Indianapolis, IN).

\section{Subclinical Atherosclerosis measures}

Carotid intima media thickness: High-resolution B-mode ultrasonography was conducted for measurement of right and left internal and common carotid artery intima-media thickness (cIMT). Complete details of the protocol have been published and vascular technicians at both study sites were trained and certified on the scanning protocol by the reading center [17]. Briefly, the vascular technician located the bifurcation of the carotid artery, and identified the maximal wall thickening in the near or far wall, in the carotid bulb or internal carotid artery. We measured maximum cIMT in each of 8 arterial segments from each side: the near and far wall images were analyzed from the lateral view of the common carotid artery and the anterior, lateral and posterior views of the bifurcation or the internal carotid artery. Each of these images was collected in a specified order and recorded and the digitized data were mailed to the Ward A. Riley Ultrasound Reading Center at Wake Forest School of Medicine for wall-thickness measurements. The maximum CIMT of the far wall for each of the segments was measured using standardized protocol.

Coronary artery calcium Agatston scores: Cardiac CT scans were performed using a gated-cardiac CT scanner, as previously described [17]. Participants were examined in the supine position and a scout image was taken to determine the level of the carina and the first scan was set $1 \mathrm{~cm}$ below the carina. Scanning was performed from superior to inferior, and a total of 46 images were obtained with 3.0-mm slice thickness. All CT scans were sent in batches to the Reading Center at Harbor-UCLA Medical Center, where they were read with Rephot Imaging software according to published methods [24]. Coronary artery calcium Agatston scores were reported for each of the four major coronary arteries and the summed score was used.

\section{Statistical Analyses}

Baseline characteristics were compared using $t$-tests for continuous variables and chi-squared tests of homogeneity for categorical variables. We checked the correlation between the continuous duration of residence in the U.S. variable with the traditional cultural beliefs variable using Pearson correlation.

We used a continuous outcome measure for CIMT and a categorical measure for CAC given the highly skewed distribution of CAC. Linear regression models were used to examine the unadjusted association between the acculturation predictors, covariates, and the continuous cIMT outcomes; results are presented as betas (change in mean cIMT) with 95\% confidence intervals (CIs) for the given unit of change in the predictor or covariate. Multinomial logistic regression models were used to examine the unadjusted association between the acculturation predictors, covariates, and the categorical CAC outcome (CAC $=0$ [referent], 1-400, and $>400$ ), with results presented as relative risk ratios (RRR) and 95\% CIs.

Linear regression (cIMT outcomes) and multinomial logistic regression (categorical CAC outcome) were used to determine which acculturation measures were significantly associated with the atherosclerosis outcomes at $p<0.20$ after adjustment for age, sex, and site which produced the "acculturation model". We determined the age, sex, and site-adjusted association between each significant 
acculturation measure and each potential mediator. Mediators which were associated with at least one of the acculturation measures at $\mathrm{p}<0.20$ were entered into a full multivariable model. We tested the interaction between sex and significant acculturation measures prior to adding the mediators to determine whether the effect of acculturation on atherosclerosis outcomes differed for men and women.

All analyses were conducted in SAS version 9.3 (SAS Institute, Cary, NC) and Stata version 12.1 (Stata Corporation, College Station, TX).

\section{Results}

Of the 906 participants enrolled in the MASALA study, a total of 900 had cIMT measures and from this subgroup, a total of 894 had available CAC measures. Table 1 shows the acculturation and atherosclerosis characteristics of the participants. Approximately $98 \%$ of participants were foreign-born and had lived in the U.S. for an average of 27 years. While there was no difference in the mean years or percent of life lived in the U.S. by sex, more women spoke English poorly or not at all compared to men. Significantly more men reported eating only or mostly South Asian food at home, shopping more frequently at South Asian markets, and having only or mostly South Asian friends than women. The median score on the traditional cultural beliefs scale did not differ by sex, but was slightly more skewed to the right (weaker beliefs) among women than men. Mean cIMT values and median CAC scores were significantly higher for men than women.

\begin{tabular}{|l|c|}
\hline & Overall $\mathbf{n = 9 0 0}$ \\
\hline Age, years & $55 \pm 9$ \\
\hline NWU study site & $410(46)$ \\
\hline Years lived in the U.S. & $27 \pm 11$ \\
\hline Percent of life in the U.S. & $50 \pm 18$ \\
\hline Speaks English poorly or not at all & $31(3)$ \\
\hline Food eaten at home: & \\
\hline Mostly/only other types of foods & $66(7)$ \\
\hline Equally South Asian/other & $354(39)$ \\
\hline Mostly/only South Asian & $480(53)$ \\
\hline Food eaten at restaurants: & $369(41)$ \\
\hline Mostly/only other types of foods & $375(42)$ \\
\hline Equally South Asian/other & $156(17)$ \\
\hline Mostly/only South Asian & \\
\hline Frequency of eating out at social/cultural/religious \\
events:
\end{tabular}




\begin{tabular}{|c|c|}
\hline Common cIMT, mm & $0.88 \pm 0.23$ \\
\hline Internal cIMT, mm & $1.21 \pm 0.45$ \\
\hline CAC score & $0(0-45)$ \\
\hline CAC score category: & \\
\hline 0 & $517(58)$ \\
\hline $1-400$ & $313(35)$ \\
\hline$>400$ & $64(7)$ \\
\hline
\end{tabular}

n (\%) or mean \pm SD is displayed; median (interquartile range) is shown for skewed variables

Table 1: Baseline characteristics of the MASALA study participants, 2010-2013*

Table 2 shows the bivariate results for each of the two continuous cIMT outcomes and the categorical CAC outcome with the acculturation measures and other potential socioeconomic, lifestyle and cardiovascular disease risk factors. More years of residence in the U.S. (or percent life in the U.S.) was associated with higher cIMT and CAC values. Other proxies of acculturation, such as language proficiency and friend and food preferences, did not have strong associations with atherosclerosis. Those who ate South Asian food equally as much as other food in restaurants had somewhat higher cIMT values than those eating either just South Asian or other foods in restaurants. Having traditional cultural beliefs in the moderate range was associated with lower common cIMT levels than having weaker beliefs. There was also a trend for stronger traditional cultural beliefs to be associated with higher odds of CAC in the moderate range (1-400 score). The correlation between years of residence in the U.S. and the traditional cultural beliefs score was low (Pearson $\mathrm{r}=0.22$ ).

\begin{tabular}{|c|c|c|c|c|c|c|c|c|}
\hline & \multirow{2}{*}{\multicolumn{2}{|c|}{ Common Carotid IMT ${ }^{*}$}} & \multirow{2}{*}{\multicolumn{2}{|c|}{ Internal Carotid IMT* }} & \multicolumn{4}{|c|}{ CAC category (ref. CAC $=0$ ) } \\
\hline & & & & & \multicolumn{2}{|c|}{ CAC $1-400$} & \multicolumn{2}{|c|}{ CAC $>400$} \\
\hline & $\beta(95 \% \mathrm{CI})$ & $p$ & $\beta(95 \% \mathrm{CI})$ & $p$ & RRR (95\% CI) & $p$ & RRR (95\% CI) & $p$ \\
\hline Age, per 10 years & $0.10(0.09-0.12)$ & $<0.001$ & $0.20(0.17-0.23)$ & $<0.001$ & $2.60(2.17-3.12)$ & $<0.001$ & $7.04(4.85-10.22)$ & $<0.001$ \\
\hline Male sex & $0.08(0.05-0.11)$ & $<0.001$ & $0.13(0.07-0.18)$ & $<0.001$ & $3.96(2.93-5.36)$ & $<0.001$ & $7.70(3.93-15.09)$ & $<0.001$ \\
\hline NWU study site & $-0.12(-0.15$ to -0.09$)$ & $<0.001$ & $-0.21(-0.27$ to -0.15$)$ & $<0.001$ & $1.37(1.03-1.82)$ & 0.03 & $0.91(0.54-1.54)$ & 0.72 \\
\hline $\begin{array}{l}\text { Years in the U.S., } \\
\text { per year }\end{array}$ & $0.004(0.003-0.005)$ & $<0.001$ & $0.008(0.005-0.01)$ & $<0.001$ & $1.04(1.03-1.06)$ & $<0.001$ & $1.09(1.06-1.12)$ & $<0.001$ \\
\hline \multicolumn{9}{|l|}{$\begin{array}{l}\text { Percent of life in } \\
\text { the U.S.: }\end{array}$} \\
\hline$\geq 80 \%$ & - & & - & & - & $0.01 \dagger$ & - & $0.008 \dagger$ \\
\hline $60-79 \%$ & $0.13(0.06-0.21)$ & $<0.001$ & $0.30(0.15-0.44)$ & $<0.001$ & - & - & - & - \\
\hline $40-59 \%$ & $0.07(0.001-0.14)$ & 0.05 & $0.17(0.04-0.31)$ & 0.01 & $0.80(0.57-1.12)$ & 0.19 & $0.50(0.28-0.91)$ & 0.02 \\
\hline $20-39 \%$ & $0.07(-0.001$ to 0.15$)$ & 0.05 & $0.10(-0.04$ to 0.25$)$ & 0.16 & $0.55(0.36-0.84)$ & 0.005 & $0.34(0.15-0.76)$ & 0.009 \\
\hline $0-19 \%$ & $0.09(0.002-0.18)$ & 0.04 & $0.25(0.07-0.42)$ & 0.006 & $0.68(0.36-1.28)$ & 0.23 & $0.41(0.12-1.44)$ & 0.16 \\
\hline $\begin{array}{l}\text { Speaks English } \\
\text { poorly/not at all }\end{array}$ & $0.04(-0.04$ to 0.13$)$ & 0.30 & $0.11(-0.05$ to 0.27$)$ & 0.17 & $0.78(0.35-1.74)$ & 0.54 & $1.29(0.37-4.48)$ & 0.69 \\
\hline \multicolumn{9}{|l|}{$\begin{array}{l}\text { Food eaten at } \\
\text { home: }\end{array}$} \\
\hline Mostly/only other & - & - & - & - & - & & - & \\
\hline Equally SA/other & $-0.008(-0.07$ to 0.05$)$ & 0.79 & 0.05 (-0.06 to 0.17$)$ & 0.37 & $1.08(0.61-1.91)$ & 0.80 & $1.12(0.41-3.07)$ & 0.82 \\
\hline Mostly/only SA & $-0.009(-0.07$ to 0.05$)$ & 0.78 & $0.09(-0.03$ to 0.20$)$ & 0.14 & $1.23(0.70-2.16)$ & 0.46 & $0.89(0.33-2.42)$ & 0.82 \\
\hline \multicolumn{9}{|l|}{$\begin{array}{l}\text { Food eaten at } \\
\text { restaurants: }\end{array}$} \\
\hline Mostly/only other & - & - & - & - & - & & - & \\
\hline Equally SA/other & $0.03(0.001-0.07)$ & 0.04 & $0.07(0.008-0.14)$ & 0.03 & $1.11(0.81-1.51)$ & 0.51 & $1.11(0.64-1.94)$ & 0.72 \\
\hline Mostly/only SA & $0.01(-0.03$ to 0.06$)$ & 0.49 & $0.04(-0.04$ to 0.12$)$ & 0.34 & $1.34(0.90-1.99)$ & 0.15 & $0.78(0.34-1.78)$ & 0.55 \\
\hline \multicolumn{9}{|l|}{$\begin{array}{l}\text { Frequency of eat- } \\
\text { ing out at social/ } \\
\text { cultural/religious } \\
\text { events: }\end{array}$} \\
\hline$<$ Once per week & - & - & - & $0.03 \dagger$ & - & & - & \\
\hline Once per week & $-0.02(-0.06$ to 0.008$)$ & 0.14 & $-0.04(-0.11$ to 0.02$)$ & 0.20 & $0.83(0.61-1.13)$ & 0.24 & $0.62(0.33-1.13)$ & 0.12 \\
\hline 2-3 times per week & $-0.03(-0.08$ to 0.02$)$ & 0.20 & $-0.10(-0.20$ to -0.006$)$ & 0.04 & $0.89(0.56-1.42)$ & 0.62 & $0.98(0.43-2.20)$ & 0.96 \\
\hline
\end{tabular}




\begin{tabular}{|c|c|c|c|c|c|c|c|c|}
\hline & \multirow{2}{*}{\multicolumn{2}{|c|}{ Common Carotid IMT* }} & \multirow{2}{*}{\multicolumn{2}{|c|}{ Internal Carotid IMT ${ }^{*}$}} & \multicolumn{4}{|c|}{ CAC category (ref. $\mathrm{CAC}=0$ ) } \\
\hline & & & & & \multicolumn{2}{|c|}{ CAC $1-400$} & \multicolumn{2}{|c|}{$\mathrm{CAC}>400$} \\
\hline & $\beta(95 \% \mathrm{CI})$ & $P$ & $\beta(95 \% \mathrm{CI})$ & $p$ & $\operatorname{RRR}(95 \% \mathrm{CI})$ & $p$ & $\operatorname{RRR}(95 \% \mathrm{CI})$ & $p$ \\
\hline \multicolumn{9}{|l|}{$\begin{array}{l}\text { Frequency of shop- } \\
\text { ping at South Asian } \\
\text { markets: }\end{array}$} \\
\hline $\begin{array}{l}\text { Almost never/ } \\
\text { never }\end{array}$ & - & - & - & - & - & & - & \\
\hline$<$ Once per month & $0.01(-0.11$ to 0.14$)$ & 0.81 & $0.06(-0.18$ to 0.30$)$ & 0.61 & $2.29(0.60-8.72)$ & 0.22 & $2.06(0.24-17.49)$ & 0.51 \\
\hline $\begin{array}{l}\text { Once or twice per } \\
\text { month }\end{array}$ & $0.03(-0.09$ to 0.15$)$ & 0.67 & $0.04(-0.19$ to 0.28$)$ & 0.71 & $1.93(0.53-7.09)$ & 0.32 & $1.14(0.14-9.28)$ & 0.91 \\
\hline $\begin{array}{l}\text { Once or more per } \\
\text { week }\end{array}$ & $0.03(-0.09$ to 0.14$)$ & 0.67 & $0.09(-0.14$ to 0.32$)$ & 0.45 & $2.46(0.68-8.94)$ & 0.17 & $1.36(0.17-10.92)$ & 0.77 \\
\hline $\begin{array}{l}\text { Ethnicity of } \\
\text { friends: Mostly/ } \\
\text { only other }\end{array}$ & & - & - & - & - & & - & \\
\hline Equally SA/other & $-0.004(-0.06$ to 0.05$)$ & 0.88 & $0.02(-0.09$ to 0.12$)$ & 0.75 & $1.14(0.69-1.90)$ & 0.61 & $0.96(0.39-2.37)$ & 0.94 \\
\hline Mostly/only SA & $-0.02(-0.07$ to 0.03$)$ & 0.46 & $0.03(-0.07$ to 0.13$)$ & 0.60 & $1.14(0.70-1.86)$ & 0.60 & $0.94(0.40-2.23)$ & 0.89 \\
\hline \multicolumn{9}{|l|}{$\begin{array}{l}\text { Traditional cultural } \\
\text { beliefs: }\end{array}$} \\
\hline Weak beliefs & - & - & - & - & - & $0.04 \dagger$ & - & \\
\hline Moderate beliefs & $-0.05(-0.09$ to -0.02$)$ & 0.003 & $0.01(-0.06$ to 0.08$)$ & 0.72 & $1.22(0.86-1.73)$ & 0.26 & $0.69(0.36-1.35)$ & 0.28 \\
\hline Strong beliefs & $-0.03(-0.06$ to 0.007$)$ & 0.11 & $0.01(-0.06$ to 0.08$)$ & 0.78 & $1.42(1.01-1.99)$ & 0.04 & $0.90(0.49-1.65)$ & 0.73 \\
\hline $\begin{array}{l}\text { Education, } \geq \text { Bach- } \\
\text { elor's degree }\end{array}$ & $\begin{array}{c}-0.05(-0.09 \text { to } \\
-0.002)\end{array}$ & 0.04 & $-0.08(-0.17$ to 0.01$)$ & 0.07 & $0.93(0.61-1.42)$ & 0.74 & $1.11(0.48-2.54)$ & 0.81 \\
\hline $\begin{array}{l}\text { Family income, } \\
\geq \$ 75,000\end{array}$ & $-0.03(-0.06$ to 0.006$)$ & 0.11 & $-0.07(-0.14$ to -0.004$)$ & 0.04 & $0.55(0.40-0.75)$ & $<0.001$ & $0.44(0.25-0.78)$ & 0.005 \\
\hline Current smoker & $0.09(0.01-0.18)$ & 0.03 & $0.02(-0.14$ to 0.18$)$ & 0.81 & $1.97(0.90-4.32)$ & 0.09 & $2.81(0.88-8.97)$ & 0.08 \\
\hline $\begin{array}{l}\text { Alcohol: No con- } \\
\text { sumption }\end{array}$ & - & $<0.001 \dagger$ & - & - & - & $0.02 \dagger$ & - & $0.04 \dagger$ \\
\hline 1-7 drinks/week & $0.04(0.005-0.07)$ & 0.02 & $0.01(-0.05$ to 0.08$)$ & 0.66 & $1.24(0.91-1.70)$ & 0.18 & $1.45(0.82-2.56)$ & 0.20 \\
\hline$>7$ drinks/week & $0.16(0.09-0.24)$ & $<0.001$ & $0.16(0.02-0.30)$ & 0.03 & $2.28(1.15-4.50)$ & 0.02 & $2.98(1.04-8.60)$ & 0.04 \\
\hline $\begin{array}{l}\text { Total physical } \\
\text { activity, per -4086 } \\
\text { MET-min/wk }\end{array}$ & $0.02(0.001-0.03)$ & 0.04 & $0.02(-0.004$ to 0.05$)$ & 0.09 & $1.41(1.21-1.66)$ & $<0.001$ & $1.60(1.17-2.19)$ & 0.003 \\
\hline $\begin{array}{l}\text { Total exercise, per } \\
\text {-1329 MET-min/ } \\
\text { week }\end{array}$ & $-0.002(-0.02$ to 0.01$)$ & 0.84 & $-0.004(-0.03$ to 0.03$)$ & 0.81 & $1.02(0.89-1.18)$ & 0.76 & $1.03(0.79-1.34)$ & 0.81 \\
\hline $\begin{array}{l}\text { Total caloric intake, } \\
\text { per } 504 \mathrm{kcal} / \text { day }\end{array}$ & $0.02(0.002-0.03)$ & 0.03 & $0.005(-0.02$ to 0.03$)$ & 0.73 & $1.04(0.91-1.20)$ & 0.56 & $1.00(0.77-1.31)$ & 0.97 \\
\hline BMI, per $5 \mathrm{~kg} / \mathrm{m} 2$ & $0.03(0.01-0.04)$ & 0.003 & $0.05(0.02-0.08)$ & 0.003 & $1.12(0.95-1.32)$ & 0.18 & $1.16(0.87-1.55)$ & 0.31 \\
\hline $\begin{array}{l}\text { Waist circumfer- } \\
\text { ence, per } 10 \mathrm{~cm}\end{array}$ & $0.04(0.02-0.05)$ & $<0.001$ & $0.07(0.05-0.10)$ & $<0.001$ & $1.53(1.32-1.77)$ & $<0.001$ & $1.73(1.35-2.23)$ & $<0.001$ \\
\hline $\begin{array}{l}\text { Systolic blood pres- } \\
\text { sure, per } 10 \mathrm{mmHg}\end{array}$ & $0.04(0.03-0.05)$ & $<0.001$ & $0.06(0.04-0.08)$ & $<0.001$ & $1.35(1.22-1.48)$ & $<0.001$ & $1.67(1.43-1.95)$ & $<0.001$ \\
\hline $\begin{array}{l}\text { Diastolic blood } \\
\text { pressure, per } 10 \\
\mathrm{mmHg}\end{array}$ & $0.01(-0.001$ to 0.03$)$ & 0.07 & $-0.02(-0.05$ to 0.01$)$ & 0.22 & $1.16(1.00-1.34)$ & 0.04 & $1.28(0.99-1.67)$ & 0.06 \\
\hline $\begin{array}{l}\text { Diabetes category: } \\
\text { Normal }\end{array}$ & - & $0.004 \dagger$ & - & $<0.001 \dagger$ & - & $<0.001 \dagger$ & - & $<0.001 \dagger$ \\
\hline Pre-diabetes & $0.01(-0.03$ to 0.04$)$ & 0.60 & $0.02(-0.05$ to 0.09$)$ & 0.60 & $1.77(1.26-2.49)$ & 0.001 & $1.77(0.88-3.57)$ & 0.11 \\
\hline Diabetes & $0.06(0.02-0.10)$ & 0.003 & $0.17(0.10-0.24)$ & $<0.001$ & $3.29(2.28-4.75)$ & $<0.001$ & $5.48(2.85-10.52)$ & $<0.001$ \\
\hline $\begin{array}{l}\text { Fasting glucose, } \\
\text { per } 24 \mathrm{mg} / \mathrm{dl}\end{array}$ & $0.02(0.01-0.04)$ & 0.005 & $0.08(0.05-0.11)$ & $<0.001$ & $1.63(1.37-1.94)$ & $<0.001$ & $1.93(1.54-2.41)$ & $<0.001$ \\
\hline $\begin{array}{l}\text { Fasting insulin, per } \\
16 \mu \mathrm{U} / \mathrm{ml}\end{array}$ & $0.01(-0.003$ to 0.03$)$ & 0.11 & $0.03(0.002-0.06)$ & 0.03 & $1.26(0.99-1.59)$ & 0.06 & $1.33(1.03-1.73)$ & 0.03 \\
\hline $\begin{array}{l}\text { Total cholesterol, } \\
\text { per } 37 \mathrm{mg} / \mathrm{dl}\end{array}$ & $-0.004(-0.02$ to 0.01$)$ & 0.59 & $-0.03(-0.06$ to -0.003$)$ & 0.03 & $0.73(0.63-0.84)$ & $<0.001$ & $0.61(0.46-0.80)$ & 0.001 \\
\hline
\end{tabular}




\begin{tabular}{|c|c|c|c|c|c|c|c|c|}
\hline & \multirow{2}{*}{\multicolumn{2}{|c|}{ Common Carotid IMT* }} & \multirow{2}{*}{\multicolumn{2}{|c|}{ Internal Carotid IMT ${ }^{\star}$}} & \multicolumn{4}{|c|}{ CAC category (ref. CAC $=0$ ) } \\
\hline & & & & & \multicolumn{2}{|c|}{ CAC 1-400 } & \multicolumn{2}{|c|}{ CAC $>400$} \\
\hline & $\beta(95 \% \mathrm{CI})$ & $p$ & $\beta(95 \% \mathrm{CI})$ & $P$ & RRR (95\% CI) & $p$ & RRR (95\% CI) & $p$ \\
\hline $\begin{array}{l}\text { LDL-cholesterol, } \\
\text { per } 32 \mathrm{mg} / \mathrm{dl}\end{array}$ & $0.002(-0.01$ to 0.02$)$ & 0.79 & $-0.03(-0.06$ to 0.002$)$ & 0.07 & $0.74(0.64-0.86)$ & $<0.001$ & $0.66(0.50-0.88)$ & 0.004 \\
\hline $\begin{array}{l}\text { HDL-cholesterol, } \\
\text { per }-13 \mathrm{mg} / \mathrm{dL}\end{array}$ & $0.02(0.001-0.03)$ & 0.03 & $0.02(-0.01$ to 0.05$)$ & 0.15 & $1.25(1.08-1.45)$ & 0.003 & $1.05(0.81-1.36)$ & 0.71 \\
\hline $\begin{array}{l}\text { Triglycerides, per } \\
71 \mathrm{mg} / \mathrm{dL}\end{array}$ & $-0.005(-0.02$ to 0.01$)$ & 0.52 & $-0.001(-0.03$ to 0.03$)$ & 0.94 & $1.04(0.91-1.20)$ & 0.54 & $0.74(0.52-1.05)$ & 0.09 \\
\hline $\begin{array}{l}\text { Cholesterol med } \\
\text { use }\end{array}$ & $0.06(0.03-0.10)$ & $<0.001$ & $0.15(0.08-0.21)$ & $<0.001$ & $2.99(2.18-4.11)$ & $<0.001$ & $5.03(2.94-8.63)$ & $<0.001$ \\
\hline
\end{tabular}

mean common cIMT was $0.88 \pm 0.23$ and mean internal cIMT was $1.21 \pm 0.45 \mathrm{~mm}$

${ }^{\dagger}$ significant $\mathrm{p}$-values for trend across categories

Table 2: Bivariate association between all covariates and atherosclerosis outcomes, MASALA study, 2010-2013

Higher education was associated with lower common cIMT and higher family income was associated with lower internal cIMT and CAC. Higher BMI, waist circumference, systolic blood pressure, and fasting glucose were all associated with higher atherosclerosis measures. Use of a cholesterol lowering medication was associated with higher levels of subclinical atherosclerosis.

In the age, sex, and site-adjusted acculturation model, the only variable associated with common cIMT was the traditional cultural beliefs measure with the moderate traditional beliefs group having significantly lower cIMT than those with weaker traditional beliefs $(\beta-0.05, p=0.004)$ (Table 3). After adjusting for other cardiovascular risk factors and potential mediators between acculturation and atherosclerosis, the moderate traditional beliefs category remained significantly associated with lower common cIMT than among those with weak traditional beliefs (Table 3). There were no age, sex, and site-adjusted acculturation measures associated with internal cIMT. Only the years lived in the U.S. variable was significantly associated with the CAC outcome in the acculturation model. (Table 3) After adjusting for other risk factors and mediators, each additional year of residence in the U.S. was associated with a $2-4 \%$ higher risk of elevated CAC. These associations did not vary by sex.

\begin{tabular}{|c|c|c|c|c|c|c|}
\hline & \multirow{2}{*}{\multicolumn{2}{|c|}{ Common CIMT $^{*}$}} & \multicolumn{4}{|c|}{ CAC Category (reference $=0$ ) } \\
\hline & & & \multicolumn{2}{|c|}{ CAC 1-400 } & \multicolumn{2}{|c|}{$\mathrm{CAC}>400$} \\
\hline & $\beta(95 \% \mathrm{CI})$ & p-value & RRR (95\% CI) & $p$-value & RRR (95\% CI) & p-value \\
\hline \multicolumn{7}{|l|}{ Acculturation model: } \\
\hline \multicolumn{7}{|l|}{ Traditional cultural beliefs: Weak (ref.) } \\
\hline Moderate beliefs & $-0.05(-0.08$ to -0.02$)$ & 0.004 & - & - & - & - \\
\hline Strong beliefs & $-0.02(-0.05$ to 0.01$)$ & 0.26 & & & & \\
\hline Years lived in the US, per 1 year & - & - & $1.02(1.00-1.03)$ & 0.03 & $1.04(1.01-1.07)$ & 0.01 \\
\hline Age, per 10 years & $0.10(0.09-0.11)$ & $<0.001$ & $2.56(2.08-3.15)$ & $<0.001$ & $6.27(4.21-9.36)$ & $<0.001$ \\
\hline Male sex & $0.07(0.04-0.10)$ & $<0.001$ & $4.57(3.27-6.40)$ & $<0.001$ & $9.53(4.59-19.78)$ & $<0.001$ \\
\hline NWU study site & $-0.12(-0.15$ to -0.10$)$ & $<0.001$ & $1.26(0.91-1.74)$ & 0.16 & $0.87(0.47-1.59)$ & 0.64 \\
\hline \multicolumn{7}{|l|}{ Multivariate model: } \\
\hline \multicolumn{7}{|l|}{ Traditional cultural beliefs: Weak (ref.) } \\
\hline Moderate beliefs & $-0.04(-0.08$ to -0.01$)$ & 0.007 & - & - & - & - \\
\hline Strong beliefs & $-0.02(-0.05$ to 0.01$)$ & 0.22 & & & & \\
\hline Years lived in the US, per 1 year & - & - & $1.02(1.00-1.04)$ & 0.02 & $1.04(1.00-1.07)$ & 0.02 \\
\hline Age, per 10 years & $0.10(0.09-0.12)$ & $<0.001$ & $2.44(1.97-3.03)$ & $<0.001$ & $6.65(4.30-10.30)$ & $<0.001$ \\
\hline Male sex & $0.02(-0.006$ to 0.06$)$ & 0.12 & $4.10(2.72-6.18)$ & $<0.001$ & $11.09(4.65-26.48)$ & $<0.001$ \\
\hline NWU study site & $-0.12(-0.14$ to -0.09$)$ & $<0.001$ & $1.23(0.88-1.73)$ & 0.22 & $0.76(0.40-1.45)$ & 0.41 \\
\hline \multicolumn{7}{|l|}{ Alcohol use: None (ref.) } \\
\hline 1-7 drinks/week & $0.02(-0.009$ to 0.05$)$ & 0.16 & $0.83(0.56-1.23)$ & 0.35 & $0.74(0.36-1.52)$ & 0.41 \\
\hline$>7$ drinks/week & $0.09(0.02-0.15)$ & 0.007 & $0.88(0.38-2.03)$ & 0.77 & $0.58(0.15-2.28)$ & 0.44 \\
\hline Waist circumference, per $10 \mathrm{~cm}$ & $0.02(0.01-0.04)$ & $<0.001$ & $1.30(1.09-1.55)$ & 0.003 & $1.64(1.17-2.29)$ & 0.004 \\
\hline HDL-cholesterol, per - 13 mg/dl & $0.01(-0.0009$ to 0.03$)$ & 0.07 & $1.04(0.85-1.26)$ & 0.71 & $0.79(0.55-1.13)$ & 0.20 \\
\hline Cholesterol medication use & $0.009(-0.02$ to 0.04$)$ & 0.54 & $2.28(1.58-3.29)$ & $<0.001$ & $3.74(1.95-7.17)$ & $<0.001$ \\
\hline Total Exercise, per -1329 MET-min/wk & $0.004(-0.01$ to 0.02$)$ & 0.59 & $1.07(0.89-1.28)$ & 0.47 & $1.23(0.89-1.70)$ & 0.21 \\
\hline Total Caloric intake, per $504 \mathrm{kcal} /$ day & $0.008(-0.005$ to 0.02$)$ & 0.22 & $0.96(0.81-1.13)$ & 0.61 & $0.85(0.60-1.19)$ & 0.33 \\
\hline
\end{tabular}

Variables not included in the model are noted with a - mark

*mean common cIMT was $0.88 \pm 0.23$

Table 3: Multivariate association with acculturation measures and atherosclerosis after adjusting for all covariates 


\section{Discussion}

In a community-based sample of middle-older aged South Asians, we found that a longer duration of residence in the U.S. was associated with higher levels of subclinical atherosclerosis. This direct effect of U.S. residence remained robust with coronary artery calcium after further adjustment for covariates and lifestyle mediators. Another multi-dimensional measure of acculturation developed among South Asians, strength of traditional cultural beliefs, was associated with lower common carotid intima media thickness among those with moderate traditional beliefs only. These findings highlight alternative ways to measure cultural factors in epidemiologic research and their potential influence on cardiovascular disease risk.

Several studies have examined the effect of duration of residence in the U.S. as a proxy measure for acculturation and atherosclerosis. In MESA, years of residence in the U.S. was positively associated with the presence and amount of coronary artery calcium in Chinese, African Americans, and Latinos and this association were not accounted for by smoking, BMI, LDL and HDL-cholesterol, hypertension, and diabetes [6]. Longer residence in the U.S. was also associated with more carotid plaque [7] and higher coronary and thoracic calcium among Latinos [8]. A few studies have examined the effect of duration of residence with atherosclerosis and/ or cardiovascular disease prevalence among South Asians finding similar positive associations [25-27]. In the Canadian SHARE study, longer duration of residence in Canada was independently associated with cardiovascular disease prevalence among South Asians after adjusting for conventional risk factors, novel biomarkers and carotid atherosclerosis [25]. Similarly, we found that longer residence in the U.S. was associated with higher CAC levels even after adjusting for explanatory lifestyle behaviors and other risk factors. It is possible that other unhealthy lifestyle factors that we failed to measure adequately, including psychological stressors or environmental factors, may explain this association.

An important finding of this study is that South Asians who had moderate scores on the traditional cultural beliefs scale, possibly reflecting a bicultural affiliation, had lower common carotid intima media thickness compared to those with stronger or weaker traditional beliefs. The use of the traditional cultural beliefs scale demonstrated nuances that were not apparent when using proxy measures such as duration of residence or English language proficiency. This is particularly true for the South Asians in the MASALA study because the majority had lived in the U.S. for more than 27 years and spoke English well, suggesting this group was highly acculturated. However, the cultural beliefs scale allowed us to capture a more multidimensional picture of South Asians' cultural traditions, beliefs, and identity. The concept of biculturalism, which represents affiliation with both one's heritage culture and the host culture, and was originally derived by social scientists focusing on cultural behaviors such as language use, choice of friends, media preferences [28] but has more recently included cultural practices, values, and identifications [29]. Biculturalism, which has been measured as fluency in both a native language and in English, has been associated with lower rates of obesity in Asians in California [30] and in Latinos [31,32]. However, we are not aware of other studies measuring biculturalism using multidimensional tools and atherosclerosis in South Asians or other ethnic groups. We hypothesize that healthier lifestyle behaviors retained from the native culture and adopted from the host culture account for some of this beneficial effect; however the association was not explained by our measured lifestyle factors. Additionally, attitudes towards or knowledge about disease risk and prevention in the moderate traditional cultural beliefs group may be explanatory factors which were not measured in our study.

While we had a large community-based sample of South Asians with several measures of socioeconomic status, conventional risk factors, lifestyle factors, and atherosclerosis we were limited in a single assessment of a few selected cultural factors in this cohort. Ideally, the measurement of cultural beliefs at several time intervals over the immigration time period and assessment of change would be a better method to truly capture the effect of acculturation on any outcome. The traditional cultural beliefs scale was originally developed in an Asian Indian sample of primarily Hindu and Sikh participants [15], and may not adequately measure traditional cultural beliefs in other South Asian groups or those of other religious affiliation. Moreover, the South Asians included in MASALA were recruited from two geographic locations in the U.S. and the high socioeconomic attainment of this cohort as described previously [17] limit the generalizability of these findings to all immigrant South Asians. We may have missed the full range of acculturation in our sample, for example not having enough limited English proficient participants, and therefore may have underestimated the expected associations in all U.S. South Asians.

In conclusion, longer duration of residence in the U.S. was associated with higher CAC scores and having moderate traditional beliefs was associated with lower common cIMT in South Asians. These findings support the need for better conceptualization and measurement of how migration influences cultural beliefs and practices, and their subsequent influence on health behaviors and disease risk.

\section{Acknowledgement}

The MASALA study was supported by the NIH grant \#1R01 HL093009. Data collection at UCSF was also supported by NIH/ NCRR UCSF-CTSI Grant Number UL1 RR024131. The sponsors did not play a significant role in the analysis, interpretation, and presentation of these results.

\section{References}

1. U.S. Census Bureau (2010) 2010 Census--Demographic Profile Data.

2. Gordon MM (1964) Assimilation in American life: The role of race, religion and national origins. Oxford University, New York. 
3. Clark L, Hofsess L (1998) Acculturation. Handbook of Immigrant Health. New York, NY: Plenum Press: 37-59.

4. Robertson TL, Kato H, Gordon T, Kagan A, Rhoads GG, et al. (1977) Epidemiologic studies of coronary heart disease and stroke in Japanese men living in Japan, Hawaii and California. Coronary heart disease risk factors in Japan and Hawaii. Am J Cardiol 39: 239-43.

5. Gordon-Larsen P, Harris KM, Ward DS, Popkin BM, National Longitudinal Study of Adolescent H (2003) Acculturation and overweight-related behaviors among Hispanic immigrants to the US: the National Longitudinal Study of Adolescent Health. Soc Sci Med 57: 2023-34.

6. Diez Roux AV, Detrano R, Jackson S, Jacobs DR, Schreiner PJ, et al. (2005) Acculturation and socioeconomic position as predictors of coronary calcification in a multiethnic sample. Circulation 112: 1557-65.

7. Lutsey PL, Diez Roux AV, Jacobs DR, Burke GL, Harman J, et al. (2008) Associations of acculturation and socioeconomic status with subclinical cardiovascular disease in the multi-ethnic study of atherosclerosis. Am J Public Health 98: 1963-70.

8. Gallo LC, de Los Monteros KE, Allison M, Diez Roux A, Polak JF, et al. (2009) Do socioeconomic gradients in subclinical atherosclerosis vary according to acculturation level? Analyses of Mexican-Americans in the multi-ethnic study of atherosclerosis. Psychosom Med 71: 756-62.

9. Judith M. Brown (2006) Global South Asians: Introducing the modern diaspora. New approaches to Asian history. J Econ Soc Hist Orient 50: 577-9.

10. Dasgupta S (1998) Gender roles and cultural continuity in the Asian Indian immigrant community in the US. Sex Roles 38: 953-74.

11. Shaw A (2000) Kinship and Continuity: Pakistani Families in Britain. Amsterdam: Harwood Acad.

12. Shankar S (2008) Desi land: teen culture, class, and success in Silicon Valley. Durham, N.C, Duke University Press.

13. Wakil SP, Siddique CM, Wakil FA (1981) Between 2 Cultures - a Study in Socialization of Children of Immigrants. J Marriage Fam 43: 929-40.

14. Suinn RM, Ahuna C, Khoo G (1992) The Suinn-Lew Asian Self-Identity Acculturation Scale: Concurrent and factorial validity. Educ Psych Measurement 52: 1041-6.

15. Mukherjea A, Underwood KC, Stewart AL, Ivey SL, Kanaya AM (2013) Asian Indian views on diet and health in the United States: importance of understanding cultural and social factors to address disparities. Fam Community Health 36: 311-23.

16. Kanaya AM, Wassel CL, Mathur D, Stewart A, Herrington D, et al. (2010) Prevalence and correlates of diabetes in South asian indians in the United States: findings from the metabolic syndrome and atherosclerosis in South asians living in america study and the multi-ethnic study of atherosclerosis. Metab Syndr Relat Disord 8: 157-64.

17. Kanaya AM, Kandula N, Herrington D, Budoff MJ, Hulley S, et al. (2013) Mediators of Atherosclerosis in South Asians Living in America (MASALA) Study: Objectives, Methods, and Cohort Description. Clin Cardiol 36: 713-20.

18. Bild DE, Bluemke DA, Burke GL, Detrano R, Diez Roux AV, et al. (2002) Multi-ethnic study of atherosclerosis: objectives and design. Am J Epidemiol 156: 871-81.

19. Kanaya AM, Kandula NR, Ewing SK, Herrington D, Liu K, et al. (2014) Comparing coronary artery calcium among U.S. South Asians with four racial/ethnic groups: The MASALA and MESA studies. Atherosclerosis 234: 102-7.

20. Kelemen LE, Anand SS, Vuksan V, Yi Q, Teo KK, et al. (2003) Development and evaluation of cultural food frequency questionnaires for South Asians, Chinese, and Europeans in North America. J Am Diet Assoc 103: 1178-84.

21. Ainsworth BE, Irwin ML, Addy CL, Whitt MC, Stolarczyk LM (1999) Moderate physical activity patterns of minority women: the Cross-Cultural Activity Participation Study. J Womens Health Gend Based Med 8: 805-13.

22. Friedewald WT, Levy RI, Fredrickson DS (1972) Estimation of the concentration of low-density lipoprotein cholesterol in plasma, without use of the preparative ultracentrifuge. Clinical Chemistry 18: 499-502.

23. ADA (2004) Diagnosis and classification of diabetes mellitus. Diabetes Care 27 Suppl 1: S5-10.

24. Carr JJ, Nelson JC, Wong ND, McNitt-Gray M, Arad Y, et al. (2005) Calcified coronary artery plaque measurement with cardiac CT in population-based studies: standardized protocol of Multi-Ethnic Study of Atherosclerosis (MESA) and Coronary Artery Risk Development in Young Adults (CARDIA) study. Radiology 234: $35-43$.

25. Anand SS, Yusuf S, Vuksan V, Devanesen S, Teo KK, et al. (2000) Differences in risk factors, atherosclerosis, and cardiovascular disease between ethnic groups in Canada: the Study of Health Assessment and Risk in Ethnic groups (SHARE). Lancet 356: 279-84.

26. Mooteri SN, Petersen F, Dagubati R, Pai RG (2004) Duration of residence in the United States as a new risk factor for coronary artery disease (The Konkani Heart Study). Am J Cardiol 93: 359-61.

27. Dodani S, Dong L (2011) Acculturation, coronary artery disease and carotid intima media thickness in South Asian immigrants--unique population with increased risk. Ethn Dis 21: 314-21.

28. Berry JW (1997) Immigration, acculturation, and adaptation. Applied Psychology: An International Review 46: 5-34.

29. Schwartz SJ, Unger JB, Zamboanga BL, Szapocznik J (2010) Rethinking the concept of acculturation: implications for theory and research. Am Psychol 65: 237-51.

30. Wang S, Quan J, Kanaya AM, Fernandez A (2011) Asian Americans and obesity in California: a protective effect of biculturalism. J Immigr Minor Health 13: 276-83.

31. Khan LK, Sobal J, Martorell R (1997) Acculturation, socioeconomic status, and obesity in Mexican Americans, Cuban Americans, and Puerto Ricans. Int J Obes Relat Metab Disord 21: 91-6.

32. Sundquist J, Winkleby M (2000) Country of birth, acculturation status and abdominal obesity in a national sample of Mexican-American women and men. Int J Epidemiol 29: 470-7 


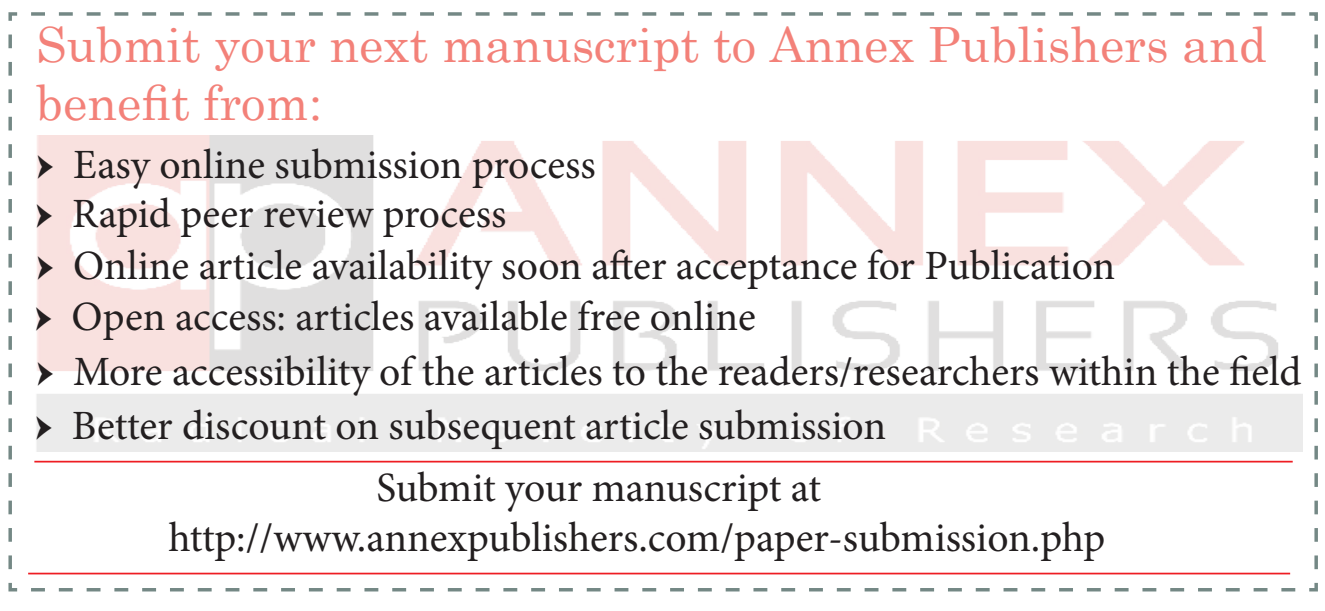

